\title{
On Application of Modified Lagrange Multipliers for Solving Optimization Problems in both Equality and Inequality Constraints
}

\author{
${ }^{1}$ S. A. Olorunsola, ${ }^{2}$ T. E. Olaosebikan And ${ }^{3}$ K. J. Adebayo \\ ${ }^{1,2,3}$ Department of Mathematical Sciences, Ekiti State University, Ado Ekiti, Nigeria.
}

\begin{abstract}
This paper discusses the application of a Modified Lagrange Multipliers Method (MLM) in solving optimization problems with equality and inequality constraints. The method is aimed at circumventing the computational rigours undergone using the Lagrange multipliers method in solving this class of problems with equality and inequality constraints independently. Also, it aims at reducing the computer memory space occupied by the independent methods in solving these problems using the said methods.

In other to achieve this, a straightforward and simple to handle MLM algorithm has been developed to solve the same optimization problems with both equality and inequality constraints. Comparing the numerical results with that of the classical methods show some improvements.
\end{abstract}

Keywords: Lagrange Multipliers, Modified Lagrange Multipliers, Optimization Problems with Equality Constraints, Optimization Problems with Inequality Constraints.

\section{Introduction}

The general optimization problem to be considered is of the form [1] and [2] as:

Optimize:

$f(x)$

1.1

Subject to:

$$
\begin{aligned}
h_{i}(x) & =0 i=1,2, \ldots, m_{1} \\
g_{j}(x) & \geq 0 j=1,2, \ldots, m_{2} \\
x & \geq 0
\end{aligned}
$$

where $x \in R^{n}, h_{i}(x)$, a vector equations of dimension $m_{1}$, and $g_{j}(x)$ is a vector of inequality of dimension $m_{2}$, such that the sum of the constraints $m=\left(m_{1}+m_{2}\right)$. The functions $f(x), h_{i}(x)$ and $g_{j}(x)$ are differentiable functions. Methods for solving this model have been developed, tested and successfully applied to many important problems of scientific and economic interest. However, in spite of the proliferation of the methods, there is no universal method for solving all optimization problems which calls for application of MLM for solving optimization problems that comprises of the equality and inequality constraints.

\section{Computational Procedure of the MLM Algorithm}

Before discussing the computational procedure of the modified Lagrange multipliers to solving this kind of optimization problems which is our major concern, it is expedient to briefly discuss how the equality and inequality type of the said problems have been solved independently with some supporting theorems which are advantageous in other to get the clear picture of what the analysis is all about and basis for the MLM algorithm.

\section{Theorem 2.1:}

Let $f$ and $g_{i} \in C^{1}$, and given the existence of the Lagrange multiplier $\lambda^{*}$, then the following conditions have to be satisfied at the point $x^{*}$ that corresponds to the solution of the primal problem:

$$
\left.\begin{array}{rl}
\frac{\delta f}{\delta x_{j}}\left(x^{*}\right)+\sum_{i=1}^{m} \lambda^{*} \frac{\delta g_{i}}{\delta x_{j}} & =0, j=1,2, \ldots, n \\
g_{i}\left(x^{*}\right) \leq 0, & i=1,2, \ldots, m \\
\lambda^{*} g_{i}\left(x^{*}\right)=0, \quad i & =1,2, \ldots, m \\
\lambda_{i}{ }^{*} \geq 0, & i=1,2, \ldots, m
\end{array}\right\}
$$

Or in a more compact notation form:

$$
\begin{aligned}
\nabla_{x} L\left(x^{*}, \lambda^{*}\right) & =0 \\
\nabla_{\lambda} L\left(x^{*}, \lambda^{*}\right) & \leq 0 \\
\lambda^{* T} g\left(x^{*}\right) & =0 \\
\lambda^{*} & \geq 0 .
\end{aligned}
$$

These conditions are known as the Karush-Kuhn-Tucker (KKT) stationary conditions [3]. 


\section{Proof:}

First convert the inequality constraints into equality constraints by introducing the slack variables $s_{i}$ :

$g_{i}(x)+s_{i}=0, i=1,2, \ldots, m$

$s_{i} \geq 0$.

Define the corresponding Lagrange function as:

$L(x, s, \lambda)=f(x)+\sum_{i=1}^{m} \lambda_{i}\left(g_{i}(x)+s_{i}\right)$.

Assume that the solution (1.1), (1.2), (1.4) with the constraints (2.2) is given by $x^{*}, s^{*}$.

Now distinguish between the two possibilities:

(i) Let $s_{i}{ }^{*}>0$ for all $i$. In the case the problem is identical to the usual minimization problem with equality constraints which is solved using Lagrange multipliers. Here there are $m$ additional variables

$s_{1}, s_{2}, \ldots, s_{m}$. Hence the necessary conditions for the minimum are:

$\frac{\delta L}{\delta x_{j}}\left(x^{*}, s^{*}, \lambda^{*}\right)=\frac{\delta f}{\delta x_{j}}\left(x^{*}\right)+\sum_{i=0}^{m} \lambda_{i} \frac{\delta g_{i}}{\delta x_{j}}\left(x^{*}\right)=0, j=1,2, \ldots, n$

$\frac{\delta L}{\delta s_{i}}\left(x^{*}, s^{*}, \lambda^{*}\right)=\lambda_{i}^{*}=0, i=1,2, \ldots, m$.

As $\lambda_{i}^{*}>0$, it also follows that $g_{i}\left(x^{*}\right)<0$ and with the fact that $\lambda_{i}^{*}=0$ this yields:

$\lambda_{i}{ }^{*} g_{i}\left(x^{*}\right)=0 . i=1,2, \ldots, m$.

Consequently all the conditions of the theorem hold for the case $s_{i}{ }^{*}>0$ for all $i$.

(i) Let $s_{i}{ }^{*}=0$ for $i=1,2, \ldots, p$ and $s_{i}{ }^{*}>0, i=p+1, \ldots, m$.

In this case, the solution may be considered to the solution of an equivalent minimization problem with the following equality constraints:

$$
g_{i}(x)=0, i=1,2, \ldots, p
$$

$g_{i}(x)+s_{i}=0, i=p+1, \ldots, m$.

Applying (2.4), (2.5) and (2.7) it follows that (2.6).

Obviously,

$g_{i}\left(x^{*}\right)<0, \mathrm{i}=\mathrm{p}+1, \ldots, \mathrm{m}$

And from (2.7) it follows that:

$g_{i}\left(x^{*}\right) \leq 0, \mathrm{i}=1,2, \ldots, \mathrm{m}$.

However, no information concerning $\lambda_{i}{ }^{*}, i=1, \ldots, p$ is available. This is obtained from the following additional argument. Consider feasible charges from $x^{*}, s^{*}$ in all variables $x_{1}, \ldots, x_{n}, s_{1}, \ldots, s_{m}$. Again consider $m$ of these as dependent variables and the remaining $n$ as independent variables. If $p \leq n$ then, $s_{1}, \ldots, s_{p}$ can always be included in the set of independent variables. (Find $\lambda^{*}$ by putting the partial derivatives of $L$ at $x^{*}, s^{*}$ with respect to the dependent variables, equal to zero and solving for $\left.\lambda^{*}\right)$. As $d s_{i}>0\left(s_{i}{ }^{*}=0\right)$ must apply for feasible changes in the independent variables $s_{1}, \ldots, s_{p}$, it follows that in general for changes which are consistent with the equality constraints, that $d f \geq 0$ for changes involving $s_{1}, \ldots, s_{p}$. Thus if these independent variables are varied one at a time, since all the partial derivatives of $L$ with respect to the dependent variables must be equal to zero, that:

$d f=\frac{\delta L}{\delta s_{i}}\left(x^{*}, \lambda^{*}\right) d s_{i}=\lambda_{i}^{*} d s_{i} \geq 0, i=1,2, \ldots, p$.

As $d s_{i}>0$, it follows that, $\lambda_{i} \geq 0, i=1,2, \ldots, m$. Thus, since it has already been proved that $\lambda_{i}{ }^{*}=0, i=p+1, \ldots, m$, it follows that indeed $\lambda_{i} \geq 0, i=1,2, \ldots, m$. This completes the proof of the theorem.

Remark 1: Obviously, if an equality constraint, $h_{k}(x)=0$ is also prescribed explicitly, then, $s_{k}$ does not exist and nothing is known of the sign of $\lambda_{k}{ }^{*}$ as $\frac{\delta L}{\delta s_{k}}$ does not exist.

Case 1: Equality Constraints

In this case, (1.1) and (1.2) are to be considered only, the problem is to determine a point $x^{*}$ which yields a strong relative minimum or maximum for (1.1) and also satisfies (1.2) and to achieve this using Lagrange method is by appending (1.2) to (1.1) with Lagrange multipliers:

$\lambda_{i}, i=1,2, \ldots, m$.

The new (1.1) now becomes:

$L(x, \lambda)$

where (2.13) is called Lagrangian. Now, (1.1) and (2.13) becomes:

$L(x, \lambda)=f(x)+\sum_{i=1}^{m} \lambda_{i} h_{i}(x)$

(2.14) is called Lagrangian function which is now unconstrained. We now state the necessary conditions for stationarity as follows: 
$\frac{\delta L}{\delta x_{j}}=\frac{\delta f}{\delta x_{j}}+\sum_{i=0}^{m} \lambda_{i} \frac{\delta h_{i}}{\delta x_{j}}=0, j=1,2, \ldots, n$,

$\frac{\delta L}{\delta \lambda_{i}}=h_{i}(x)=0, i=1,2, \ldots, m$,

$\left(x^{*}, \lambda^{*}\right)$

(2.15) and (2.16) yield a set of $m+n$ equations in $m+n$ unknowns $(x, \lambda)$ to be solved for the (2.17).

$L\left(x^{*}, \lambda^{*}\right)$

Remark 2: The condition (2.16) guarantees that (1.2) is satisfied at (2.18).

$f\left(x^{*}\right)$

where (2.17), (2.18) and (2.19) are optimal value, optimal solution and optimal returns respectively. In this case, (1.2) and (2.17) of (2.14) is equal to (2.19) for (1.1).

Case 2: Inequality constraint

In this case, (1.1) and (1.3) are to be considered only. Here, a real-valued slack variable $\theta_{j}$ is been defined for each (1.3) such that:

$\theta_{j}^{2}=g_{j}(x) \geq 0, j=1,2, \ldots, r$

(1.3) is satisfied by satisfying (2.20) for all real-valued $\theta_{j}$. Applying the Lagrange necessary conditions by [4] and [5], we obtain:

$$
\left.\begin{array}{c}
L(x, \lambda, \theta)=f(x)+\sum_{j=0}^{m} \lambda_{j}\left(g_{j}(x)-\theta_{j}^{2}\right) \\
\frac{\delta L}{\delta x_{i}}=\frac{\delta f}{\delta x_{i}}+\sum_{j=0}^{m} \lambda_{i} \frac{\delta g_{j}}{\delta x_{i}}=0, i=1,2, \ldots, n \\
\frac{\delta L}{\delta \lambda_{i}}=g_{j}(x)-\theta_{j}^{2}=0, j=1,2, \ldots, m, \\
\frac{\delta L}{\delta \theta_{j}}=-2 \lambda_{j} \theta_{j}=0, j=1,2, \ldots, m .
\end{array}\right\}
$$

From (2.21), either $\lambda_{j}^{*}=0$ or $\theta_{j}^{*}=0$ or both.

Having gotten the clear knowledge of independent solution of this class of problems, we now want to see if giving a problem that comprises both cases discussed earlier in this paper, can be solved using the same method. Base on this, we state the MLM algorithm and procedure in the next section.

\section{The MLM Algorithm for solving Equality and Inequality Optimization Problems}

Step 1: Change the inequality constraints into equality by introduction slack variables as in (2.20)

Step 2: Equate the equality constraints to zero.

Step 3: Introduce Lagrange multipliers as in (2.12)

Step 4: Formulate Lagrangian function as in (2.14)

Step 5: Apply the Lagrange multiplier method's necessary conditions (2.21) to the formulated Lagrange in step 4.

Step 7: Hence, determine the optimal value, optimal solution and optimal function return as in (2.17), (2.18) and (2.19) respectively.

Remark 3: (1) The slack variable can either tend to or equal to zero. Also, either addition or subtraction can be used when converting the constrained to unconstrained problem by the Lagrange multiplier.

\section{Computational Results}

The following problems were evaluated using the MLM algorithm thus:

\section{Problem (P1):}

Maximize $f(x)=7 x_{1}^{2}+6 x_{1}+5 x_{2}^{2}$,

Subject to: $\quad x_{1}+2 x_{2} \leq 10$,

$x_{1}-3 x_{2}=9$,

Problem (P2):

$$
x_{1}, x_{2} \geq 0 \text {. }
$$

Maximize $f(x)=2 x_{1}-x_{1}^{2}+x_{2}$,

Subject to: $\quad 2 x_{1}+3 x_{2}=6$,

$2 x_{1}+x_{2} \leq 4$,

$x_{1}, x_{2} \geq 0$. 
Problem (P3):

Minimize $f(x)=2 x_{1}+3 x_{2}-\left(x_{1}^{2}+x_{2}^{2}+x_{3}^{2}\right)$,

Subject to: $\quad x_{1}+x_{2} \leq 1$,

$2 x_{1}+3 x_{2}=6$,

$$
x_{1}, x_{1} \geq 0 \text {. }
$$

Problem (P4):

Minimize $f(x)=4 x_{1}+9 x_{2}-x_{1}^{2}-x_{2}^{2}$,

Subject to: $\quad 4 x_{1}+3 x_{2}=15$,

$$
3 x_{1}+5 x_{2} \geq 14
$$

$$
x_{1}, x_{2} \geq 0 \text {. }
$$

Problem (P5):

Minimize $f(x)=4 x_{1}^{2}+2 x_{2}^{2}+x_{3}^{2}-4 x_{1} x_{2}$,

Subject to: $\quad x_{1}+x_{2}+x_{3} \geq 15$,

$2 x_{1}-x_{2}+2 x_{3}=20$,

$x_{1}, x_{2}, x_{3} \geq 0$.

Problem (P6):

Minimize $f(x)=x_{1}^{2}+x_{2}^{2}+x_{3}^{2}$,

Subject to: $\quad x_{1}+x_{2}+3 x_{3} \leq 2$,

$5 x_{1}+2 x_{2}+x_{3}=5$

$$
x_{1}, x_{2}, x_{3} \geq 0 \text {. }
$$

\section{Conclusion}

In this paper, the algorithm developed has been tested on some problems with corresponding results shown in Table1 below. From the table, problem 1 with numerical result 702.920 coincide with the analytical result which is 702.920, problem 2 with numerical result 1.750 is close to the analytical result which is 2.444 , problem 5 with the numerical result 32.000 is less than the analytical result which is 91.111, also, problem 6 with numerical result of 0.783 is close to the analytical result which is 0.847 . However, problem 3 and 4 with numerical results 2.309 and 22.56 are greater than the analytical results 2.125 and 11.000 respectively. This shows that the method has worked for problem 1, 2, 5, 6 and is been violated slightly by problem 3 and 4 . With these, it is invariably justified clearly that, the MLM can be used to solve optimization problems of both equality and inequality type.

\section{References}

[1]. RAO, S. S., (1978), Optimization Theory and Applications, Willy and Sons.

[2]. THOMAS, F.E., and DAVID, M.H., (2001), Optimization of Chemical Processes, McGraw Hill

[3]. IGOR GRIVA STEPHEN G and NASH ARIELA SOFER (2009), Linear and Nonlinear Optimization, George Mason University Fairfax, Virginia.

[4]. JAN A. SNYMAN (2005), Practical Mathematical Optimization, University of Pretoria, Pretoria, South Africa.

[5]. RICHARD BRONSON and GOVINDASAMA NAADIMUTHU.(2007), Operations Research, Tata McGraw-Hill Publishing Company Limited, New Delhi.

\section{Table of Results:}

Table 1:

\begin{tabular}{|l|c|c|c|c|c|c|c|c|}
\hline \multirow{2}{*}{ Problems } & \multicolumn{4}{|c|}{ Numerical Results } & \multicolumn{4}{c|}{ Analytical Results } \\
\cline { 2 - 9 } & $x_{1}$ & $x_{2}$ & $x_{3}$ & $f(x)$ & $x_{1}$ & $x_{2}$ & $x_{3}$ & $f(x)$ \\
\hline 1 & 9.600 & 0.200 & - & 702.920 & 9.600 & 0.200 & - & 702.920 \\
\hline 2 & 1.500 & 1.000 & - & 1.750 & 0.667 & 1.556 & - & 2.444 \\
\hline 3 & 0.462 & 0.693 & 0.000 & 2.309 & 0.250 & 0.750 & 0.000 & 2.125 \\
\hline 4 & 0.960 & 3.720 & - & 22.56 & 3.000 & 1.000 & - & 11.000 \\
\hline 5 & 2.000 & 0.000 & 8.000 & 32.000 & 3.667 & 3.333 & 8.000 & 91.111 \\
\hline 6 & 0.808 & 0.323 & 0.162 & 0.783 & 0.804 & 0.348 & 0.283 & 0.847 \\
\hline
\end{tabular}

